\title{
A Sequential Study of Age-Related Lipofuscin Accumulation in Hippocampus and Striate Cortex of Rats
}

\author{
Sarika Singh Kushwaha ${ }^{a, b}$ Nisha Patro $^{a}$ Ishan Kumar Patro ${ }^{a, b}$

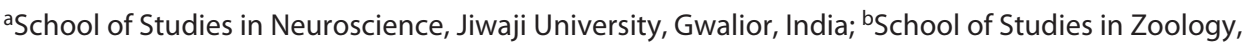 \\ Jiwaji University, Gwalior, India
}

\section{Keywords}

Lipofuscin · Hippocampus · Aging · Striate cortex · Microglia

\section{Abstract}

Background: The age-pigment, lipofuscin that accumulates in cells intrinsically and progressively with age is considered as the hallmark of aging. This accumulation is more prominent in post-mitotic cells like neurons and also appears in glia. Purpose: The aim of the present study was to assess the age-associated occurrence and distribution pattern of lipofuscin both in neurons and microglia in various regions of hippocampus and striate cortex. Methods: The accumulation pattern of lipofuscin in hippocampus and striate cortex was observed in the female Wistar rats of 6 age groups, that is, $3,6,12,18,24$, and 30 months using the autofluorescent property of lipofuscin and its specific localization in neurons and microglia by dual immunolabeling with $\mathrm{NeuN}$ and Iba-1 antibodies respectively. Cytoarchitectural and the morphological age-related changes were observed with cresyl violet staining. Results: Lipofuscin pigments accumulate progressively through the normal aging process in hippocampus and striate cortex. However, in hippocampus, lipofuscin accumulates in a region-specific manner with the highest accumulation observed in cornu ammonis (CA) 1 and 3 subregions. Furthermore, the lipofuscin accumulation was also observed in microglia in the senile brains both in the hippocampus and striate cortex. Conclusion: The progressive deposition of lipofuscin could result in cellular dysfunction. This encouraged us to forward the idea that microglia not only participate in the removal of the pigment from the neurons but also accumulate these waste products in itself. Such senescent microglia may contribute to age- related neurodegeneration as they lose the neuroprotective potential due to oxidative stress and thus may be unable to effectively phagocytose the age pigment.

(c) 2018 S. Karger AG, Basel

\section{Introduction}

Aging is a complex process related with the gradual diminution in cellular and physiological functions. The accumulation of intracellular age pigment "lipofuscin" (c) 2018 S. Karger AG, Basel

E-Mail karger@karger.com

www.karger.com/aon
Dr. Nisha Patro

School of Studies in Neuroscience

Jiwaji University

Gwalior 474011 (India)

E-Mail nishapatro@gmail.com 
appears to be the most consistent and detectable change during ageing [1-6]. Lipofuscin is also considered to be an intralysosomal "garbage" or waste material, primarily composed of $30-58 \%$ proteins, $19-51 \%$ lipid like material believed as oxidation products of polyunsaturated fatty acids and some carbohydrates and traces of metals (up to $2 \%$ ) including iron, copper, aluminium, zinc, calcium, manganese, and so on [7-9]. Lipofuscin is also not degraded by lysosomal hydrolases due to the polymerization and cross linkage of peptides with aldehydes leading to plastic-like structures impervious to biological degradation [9-11]. The un-degradable property of lipofuscin was well demonstrated by the fact that even starving cells, with activated autophagy were unable to remove the pigment [12]. The most distinctive feature of lipofuscin is autofluorescence, detected by fluorescence or laser-scanning microscopy with excitation/emission maxima of $360-420 / 540-650 \mathrm{~nm}[3,6,8]$. The color of emission varies with age (from immature pigment to mature pigment) of the individual bearing it [13]. The rate of lipofuscin accumulation has been reported to vary in different species [14], in different tissues of the same species $[14,15]$, and even different regions of the same organ [16-18], The rate of lipofuscin accumulation is high in short-lived mammals like mouse and rat as compared to long-lived species due to highly active mitochondria, which produce more superoxide and hydrogen peroxide radicals $[15,19]$.

Lipofuscin is reported to accumulate progressively in postmitotic cells such as neurons and myocardial cells [2, 9, 20-23]. The localization of lipofuscin has also been reported in glial cells, although these cells, unlike neurons, continue to divide mitotically leading to an absolute reduced accumulation of lipofuscin. Thus, the concentration of glial lipofuscin is suggested to result from the transfer of neuronal lipofuscin to glia as an attempt to participate in pigment removal rather than a high rate of intraglial lipofuscin formation [24-27]. In neurons, mature large granules of lipofuscin are located in the perinuclear area of perikaryon and also in dendrites and even in axons [27-31]. Neuronal cells with large clumps of lipofuscin usually display a large cell body with short dendrites compared to cells without or only few lipofuscin granules [32]. In early life, lipofuscin granules are small and irregularly distributed within lysosomes, but as lipofuscin accumulates over time, these granules may reach a maximum size of 3-5 $\mu \mathrm{m}$ [9]. With advancing age, pigment granules increase in size, form aggregates, and intermingle with mitochondrial profiles in the intermediate locality of the nucleus [33]. The most accepted hypothesis for lipofuscinogenesis proposed by Brunk and Terman [34], referred to as "mitochondrial-lysosomal axis theory of aging," assumes the accumulation of lipofuscin caused by incomplete degradation of damaged mitochondria by process of mitophagy. It was further supported by the presence of adenosine triphosphate synthase (ATP synthase) subunit " $c$ " in age pigment granule, which is the property of mitochondria [35]. Konig et al. [36] also reported involvement of impaired mitophagy and subsequent oxidation of mitochondrial protein in age-related lipofuscinogenesis. Previous studies have reported the gradual increase of lipofuscin in the cells of the inferior olivary nucleus and the anterior horns of the spinal cord [37], substantia nigra [38] and cerebral cortex [17, 25, 39] throughout the life, but at the end of the life span, this accumulation become accelerated. Braak [40] observed the distribution pattern of lipofuscin in various layers of visual cortex and reported pigment deposition in stellate cells of layer IVa beta and pyramidal cells of Va, while as per Peters and Sethares [41], large Meynert cells of rhesus monkey primary visual cortex accumulate a very small amount of age pigment.

The present study focuses on ways of assessing the age-associated occurrence and distribution pattern of lipofuscin both in neurons and microglia in various regions of hippocampus and striate cortex. Thus, this study would be informative to examine (1) if microglial cells accumulate the age-pigment; and (2) if there is a relationship between the amount of lipofuscin in neurons to the possible presence of such pigment in the microglia.

\section{Methods}

Experimental Animals and Tissue Processing

Female Wistar rats of various age groups $(3,6,12,18,24$, and 30 months; $n=6$ ) were used for the present study. The animals were housed in a control environment $\left(25 \pm 2{ }^{\circ} \mathrm{C}, 50 \pm\right.$ $5 \%$ humidity) with ad labitum access to standard food and water. Standard light and dark cycle of 12:12 h was strictly maintained. The use of these animals and the procedures in this study were pre-approved by the Animal Ethical Committee of Jiwaji University.

Six animals ( 3 animals for cryotomy and 3 animals for paraffin sectioning) per age group were deeply anesthetized with ether vapors and perfused transcardially with phosphate buffer saline (PBS; $\mathrm{pH}$ 7.4) containing $0.001 \%$ ethylenediaminetetraacetic acid (EDTA) followed by chilled $2 \%$ paraformaldehyde prepared in 0.01 M phosphate buffer ( $\mathrm{pH} 7.4$ ) for cryotomy or 10\% buffered formalin prepared in $0.01 \mathrm{M}$ phosphate buffer $(\mathrm{pH}$ 7.4) for paraffin sectioning. Brains were dissected out and separated at medial aspect of the cerebral hemispheres and post fixed in the respective fixative. 
For cryotomy, the tissue after post-fixation in $2 \%$ paraformaldehyde at $4{ }^{\circ} \mathrm{C}$ was washed in $0.01 \mathrm{M}$ phosphate buffer thrice (30 min in each change) and then cryoprotected with gradient concentrations of sucrose solutions $(10,20$, and $30 \%)$ prepared in $0.01 \mathrm{M}$ phosphate buffer at $4{ }^{\circ} \mathrm{C}$. Each tissue block was cut coronally into $15-\mu \mathrm{m}$ thick serial sections through the entire hippocampus and striate cortex using Leica CM1900 cryostat. The sections were picked up on chromalum gelatin-coated slides and stored at $-20^{\circ} \mathrm{C}$ to be used for immunohistochemical studies.

For paraffin sectioning, after post-fixation in $10 \%$ buffered formalin for $24 \mathrm{~h}$, the tissues were thoroughly washed with distilled water ( 3 changes of $30 \mathrm{~min}$ each) and dehydrated in graded series of ethyl alcohol, that is, 30, 50, 70, and 90\% (60 min in each grade) and absolute alcohol ( 2 changes of 90 min each). This was followed by clearing in toluene (2 changes of $45 \mathrm{~min}$ each at $37^{\circ} \mathrm{C}$ ). Subsequently, the tissues were impregnated with 2 changes $\left(4 \mathrm{~h}\right.$ each) of paraplast (Sigma; m.p. $56-58^{\circ} \mathrm{C}$ ) in a preheated incubator $\left(59.5^{\circ} \mathrm{C}\right)$. The tissue blocks were prepared with the help of Leuckhart's pieces and the coronal sections were cut serially at $10 \mu \mathrm{m}$ thickness with Leica RM 2135 rotary microtome. The sections were stretched in a water bath set at 50$52^{\circ} \mathrm{C}$ and the sections were picked up on chromalum gelatincoated slides, dried at $45^{\circ} \mathrm{C}$, and stored at $4{ }^{\circ} \mathrm{C}$ in sealed boxes till further use.

Fluorescence Imaging of Autofluorescent Lipofuscin Pigment Cryocut sections were air dried for $1 \mathrm{~h}$ at $37^{\circ} \mathrm{C}$, dehydrated in absolute alcohol ( 2 changes of $10 \mathrm{~min}$ each), cleared in xylene for $10 \mathrm{~min}$, and then mounted in distyrene tricresyl phosphate xylene (DPX). The sections were visualized with Leica DM 6000 fluorescence microscope equipped with DC 420 Digital Camera and excitation filters with transmission maximum between $370-460$ and $420-490 \mathrm{~nm}$ and barrier filter of $420 \mathrm{~nm}$. The images acquired from different areas of the hippocampus and striate cortex were finally used to calculate the area fraction of lipofuscin per frame $\left(5,981.7 \mu \mathrm{m}^{2}\right)$ by digital computer-assisted method using ImageJ software, inspired by National Institute of Health (http://rsb.info. nih.gov/ij/download.html).

\section{Dual Immunofluorescence Labeling for Localization of}

\section{Lipofuscin Pigment in Neurons and Microglia}

The air dried brain sections were washed in PBS ( 3 changes of 5 min each), followed by incubation with $1 \%$ Triton $\mathrm{X}-100$ (Sigma) in PBS for 40 min for membrane permeabilization. After washing in PBS (3 changes of 5 min each), sections were incubated with $3 \%$ normal goat serum in PBS at room temperature for $90 \mathrm{~min}$. Subsequently, the sections were incubated with a cocktail of primary antibodies; rabbit polyclonal, anti-Iba 1 (1: 300, Wako catalog No. 019-19741) for microglia and mouse monoclonal anti-NeuN (1:100, Chemicon catalog No. MAB 377 ) for neurons, diluted in $3 \%$ bovine serum albumin (BSA) in PBS overnight at $4{ }^{\circ} \mathrm{C}$. Next day, sections were brought to the room temperature, washed in PBS (3 changes of 5 min each), and incubated in cocktail of fluorochrome tagged secondary antibodies, that is, anti-rabbit FITC (1:300, Sigma) and anti-mouse TRITC (1:200, Sigma) diluted in 3\% BSA in PBS for $1 \mathrm{~h}$ at room temperature in dark. Thereafter, sections were washed in PBS (4 changes of 10 min each) in dark and mounted with Vectashield hardset mounting medium with 4'-6-diamidino-2-phenylindole

Lipofuscin in Aging Hippocampus and Striate Cortex
(DAPI, Vector). The sections were finally visualized with Leica DM 6000 fluorescence microscope equipped with DC 420 digital camera for localization of lipofuscin pigment in microglia and neurons.

\section{Cresyl Violet Staining for Histology}

To study the cytoarchitectural and the morphological changes with age, tissue sections were stained with $1 \%$ cresyl violet acetate (Sigma, C1791) prepared in acetate buffer ( $\mathrm{pH} 3.5)$ as per the Humason [42] method.

The sections from various age groups were deparaffinised in xylene ( 2 changes of $10 \mathrm{~min}$ each) and rehydrated to water through down series of alcohol, that is, 100, 90, 70, 50, and 30\% (5 min in each grade). Subsequently the sections were stained in cresyl violet solution for $2 \mathrm{~min}$, rinsed quickly in distilled water, and air dried for $45 \mathrm{~min}$ at $37^{\circ} \mathrm{C}$. The sections were dehydrated in n-butanol $(2$ changes of 2 min each), cleared in xylene, and mounted in DPX. The sections were studied under a bright field microscope for ageassociated cellular changes in hippocampus and striate cortex.

\section{Data Analysis}

One-way analysis of variance, followed by post hoc analysis with Tukey test was used to analyze the significance of data with the help of the computer-based software SigmaStat 3.5. The data was expressed as mean \pm SEM (standard error of mean). F-ratio

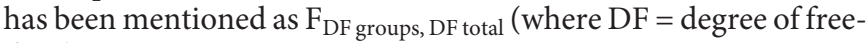
dom).

\section{Results}

\section{Lipofuscin Distribution and Processing}

With the help of the autofluorescent property of lipofuscin, the distribution pattern and rate of pigment accumulation in hippocampus and striate cortex were recorded in various age groups of Wistar rats. In 3-month old rats, only a few neurons contained sparsely scattered pigment granules, emitting whitish-yellow fluorescence, evidencing that not all the neurons start accumulating pigment simultaneously. By 6 months, more number of neurons started accumulating the pigment. However, the pigment was of the immature type and emitted a whitishyellow fluorescence (Fig. 1a, b). The pigment was found in the fine granular form and occasionally these granules fused together. By 12 and 18 months of age, the whitish yellow pigment converted into bright yellow fluorescence pigment bodies forming small clumps (Fig. 1c, d). In 24- and 30-month old animals, the intensity of autofluorescence of pigment increased immensely and shifted from yellow to bright golden yellow. The pigment was present in the form of huge clumps with greater concentration around the nucleus and often appeared like a crown on the nucleus (Fig. 1e-h). Duel immunofluorescence staining of brain sections with anti-Ibal antibody, 
Fig. 1. Photomicrographs depicting age-associated autofluorescence lipofuscin accumulation in hippocampus and striate cortex of various age groups. Immature whitish-yellow pigment in 6 months $(\mathbf{a}, \mathbf{b})$, lipofuscin granules in 12 months (c, d), bright golden-yellow, large clumps of lipofuscin in $24(\mathbf{e}, \mathbf{f})$ and 30 months $(\mathbf{g}, \mathbf{h})$. Scale bar $=20 \mu \mathrm{m}$.

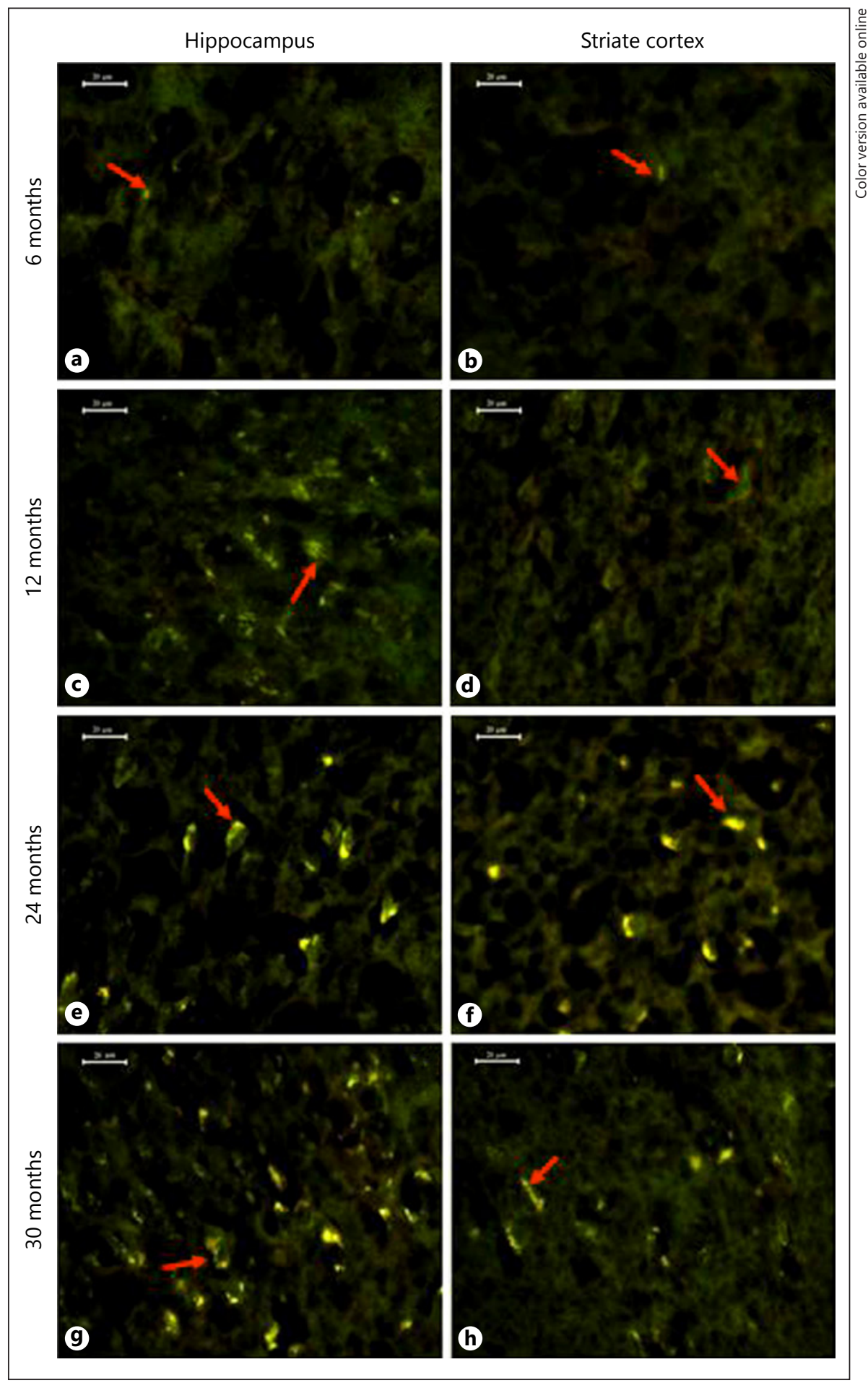

(Fig. 2b-i). At some sites, lipofuscin pigment was observed surrounded by the processes of microglia (Fig. 2a). By 18 months, microglia with degenerative processes appeared in close vicinity of lipofuscin-laden neurons in CA1 subregion (Fig. 2d-f). a microglial cell marker and anti-NeuN antibody, a neuronal marker confirmed that the microglia of 24 and 30 months old rats also contained lipofuscin pigment. These lipofuscin-laden microglia appeared in close vicinity of neurons as well as at sites far from the neurons 

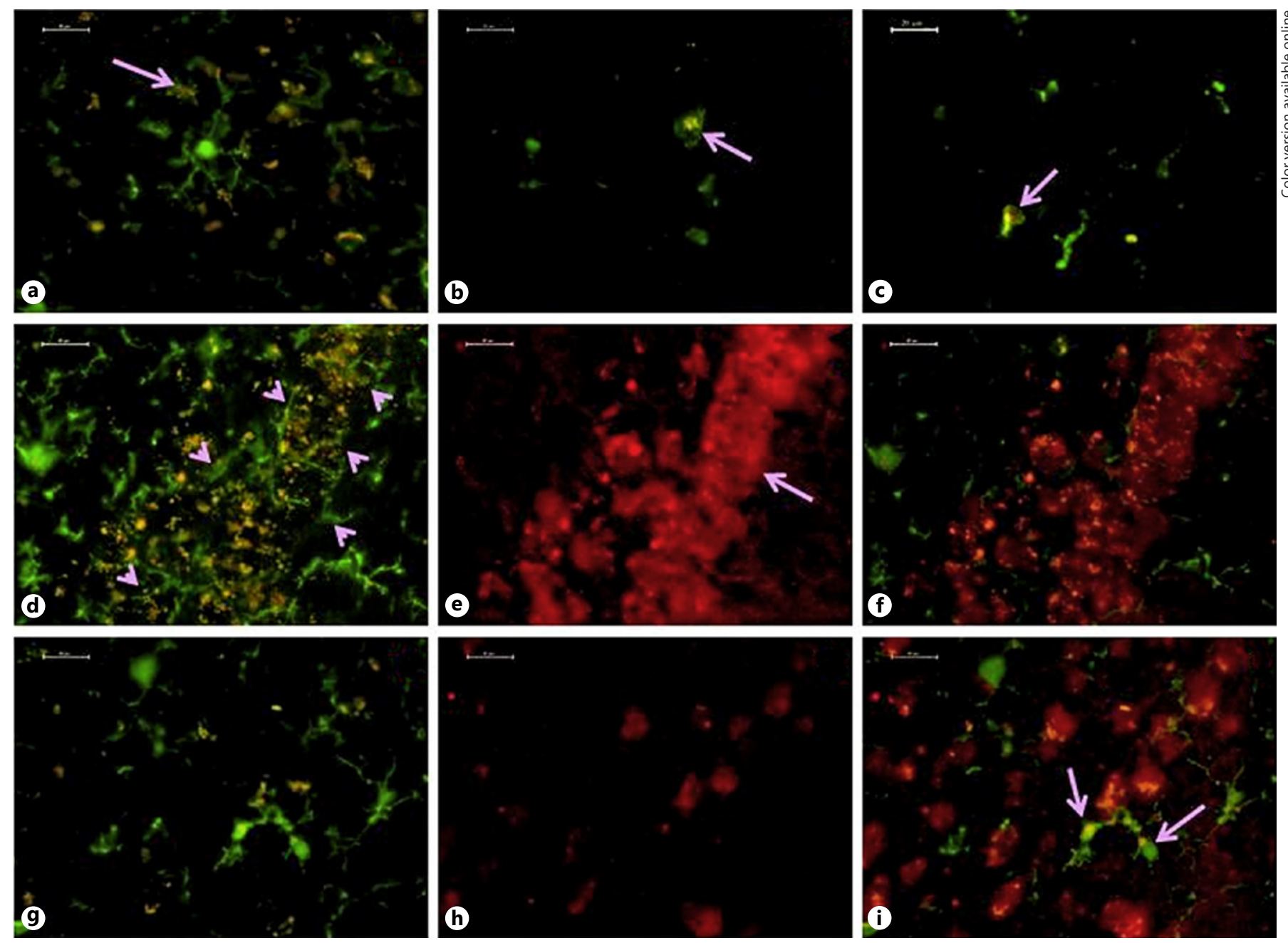

Fig. 2. Association of microglia with lipofuscin. Microglial processes surround the lipofuscin-laden neurons in hippocampus at 18 months (a; arrow). Macrophagic microglia containing pigment in striate cortex of 24 months (b; arrow) and in hippocampus of 30 months (c; arrow). Iba-1 immunolabelled microglia in the vicinity of lipofuscin-laden CA1 neurons appeared hypertrophied or with degenerating processes (d; arrowhead), NeuN labeled neu-

\section{Quantitative Changes in Lipofuscin}

The pattern of lipofuscin accumulation obtained was different in different regions of hippocampus, while the increasing pattern was almost similar all over the striate cortex. In general, irrespective of the brain area, the pigment starts accumulating after 6 months of age and subsequently, the area fraction increases linearly till old age (Fig. 3, 4). In CAl region of hippocampus, a sharp increase in lipofuscin accumulation was observed from 6 to 12 months $\left(\mathrm{F}_{[5,17]}, p<0.001\right)$ of age and a subsequent linear increase till 30 months. The accumulation remained almost constant between 12 and 18 months

Lipofuscin in Aging Hippocampus and Striate Cortex rons (e; arrow) and overlay of $(\mathbf{a}+\mathbf{b}=\mathbf{f})$ clearly shows that the Iba-1 labeled microglia with hypertrophied and degenerating processes surrounding the lipofuscin-laden CA1 neurons. Pigment present in microglia is confirmed with colocalization using Iba-1 (g) and NeuN (h) in overlay image $(\mathbf{i}=\mathbf{g}+\mathbf{h}$; arrow) at 30 months. Scale bar $=20 \mu \mathrm{m}$.

$\left(\mathrm{F}_{[5,17]}, p=0.339\right)$. Significant accumulation of pigment was observed at 18 months as compared to 3 and 6 months $\left(\mathrm{F}_{[5,17]}, p<0.001\right)$; then increased gradually till 24 months of age $\left(\mathrm{F}_{[5,17]}, p=0.002\right)$. Significant increase was also observed at 24 months as compared to 3 and 6 months $\left(\mathrm{F}_{[5,17]}, p<0.001\right)$. The area fraction of pigment was significantly high in 30 months as compared to all age groups $\left(\mathrm{F}_{[5,17]}, p<0.001\right)$. In $\mathrm{CA} 2$, the pigment accumulation also increased gradually and linearly from 6 to 30 months with a significant difference between 12 vs. 18 months $\left(\mathrm{F}_{[5,17]}, P<0.001\right), 18$ vs. 24 months $\left(\mathrm{F}_{[5,17]}, p=0.006\right)$, and 24 vs. 30 months $\left(\mathrm{F}_{[5,17]}, p=0.002\right)$. Highly significant

Ann Neurosci 2018;25:223-233 
difference was observed at 24 and 30 months as compared to $3,6,12$, and 18 months $\left(\mathrm{F}_{[5,17]}, p<0.001\right)$. Similarly in CA3 region as well, a sharp increase in lipofuscin accumulation was recorded from 6 to 12 month of age $\left(\mathrm{F}_{[5,17]}, p<0.001\right)$ and a minimum change from 12 to 18 months of age $\left(\mathrm{F}_{[5,17]}, p=0.994\right)$. Thereafter, a sharp increase in pigment accumulation was recorded from 18 to 24 months of age $\left(\mathrm{F}_{[5,17]}, p<0.001\right)$ and then a slow rate of pigment accumulation in 30 -month old rats $\left(\mathrm{F}_{[5,17]}\right.$, $p=0.201)$. This increase was significant at 24 and 30 months as compared to $3,6,12$, and 18 months $\left(\mathrm{F}_{[5}\right.$, 17], $p<0.001)$ and also at 18 and 12 months as compared to 3 and 6 months $\left(\mathrm{F}_{[5,17]}, P<0.001\right)$. In CA4 region, a sharp and steep increase was observed from 6 to 18 months and afterwards a slow but gradual increase was found till 30 months $\left(\mathrm{F}_{[5,17]}, p<0.001\right)$. A significant increase in pigment accumulation was observed at 12 months vs. 3 and 6 months $\left(\mathrm{F}_{[5,17]}, p<0.001\right)$ and 18 , 24 , and 30 months vs. 3,6 , and 12 months $\left(\mathrm{F}_{[5,17]}, p<\right.$ $0.001)$. In dentate gyrus, in contrast to other regions of the hippocampus, the rate of lipofuscin deposition was observed to be very low and significant difference was found only between 3 and 30 months $\left(\mathrm{F}_{[5,17]}, p<0.05\right)$. In general, the highest lipofuscin accumulation was found to be in CA1 and CA3 areas of the hippocampus.

In striate cortex, the pigment starts accumulating from 6 months onwards and increases significantly in a linear manner, so that the maximum pigment accumulation was observed in 30 months old rats $\left(\mathrm{F}_{[5,17]}, p<0.001\right)$. However, the pigment accumulates at a faster rate in CA1 and CA3 subfields of hippocampus than striate cortex (Fig. 5).

\section{Histological Changes in Neurons during Aging}

Brain sections stained with cresyl violet showed normal neuronal cell bodies with distinct nucleus and nucleoli and densely packed neurons in a pyramidal cell layer in the hippocampus of 3- and 6-month old rats (Fig. 6Aa13 , b1-3). By 12 months of age, the hippocampus displayed the signs of atrophy with shrunken appearance of neurons in terms of decrease in the size of perikaryon, nucleus, and nucleolus (Fig. 6Ac1-3). The degeneration was more pronounced in the CA1 subregion. Mild degeneration was observed in CA2 and CA3 subregions. DG showed minimum degeneration among all subregions. At 18 months, CA1 and CA 3 showed severe atrophy as compared to CA2 and DG subregions (Fig. 6Bd1-3). Chromatolysis with a diffused Nissl staining was observed from this age. By 18 months of age, neurons were dispersed sparsely in pyramidal cell layer (Fig. 6Bd1-3). In 24 and 30 months, CA1, CA2, and CA3 regions showed

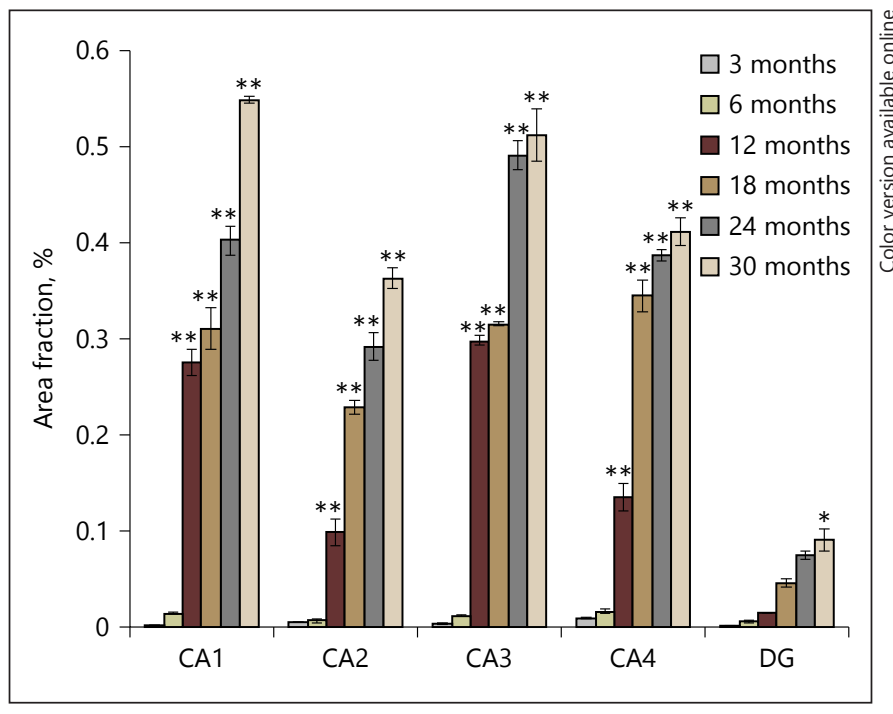

Fig. 3. Quantitative data of percent area fraction of lipofuscin accumulation in hippocampal subregions of different age groups of rat. Values are presented as mean $\pm \operatorname{SEM}(n=3) .{ }^{* *} p<0.001,{ }^{*} p<$ 0.050 for comparison of the increase in the rate of lipofuscin between various age groups.

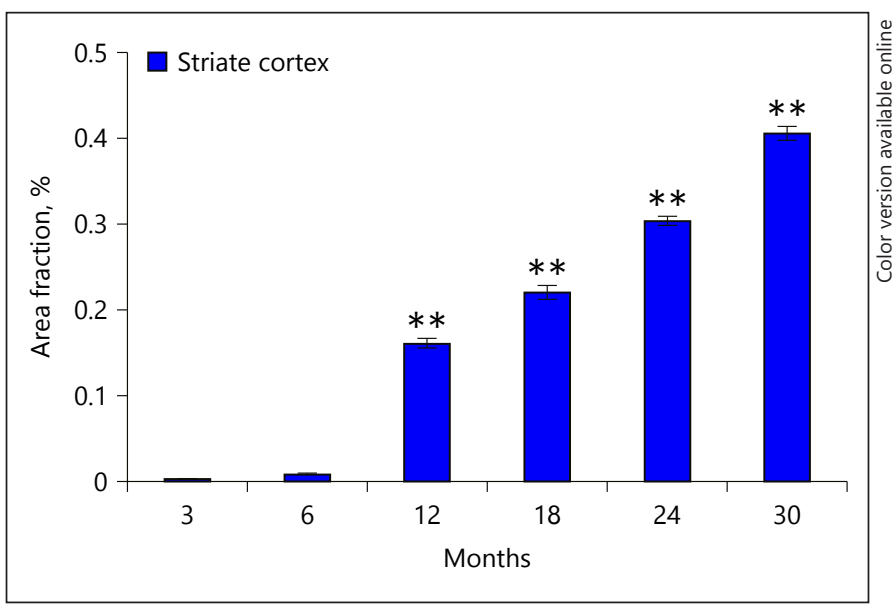

Fig. 4. Percent area fraction of lipofuscin accumulation in striate cortex of different age groups of rat. Values are presented as mean $\pm \operatorname{SEM}(n=3){ }^{* *} p<0.001$ for comparison of the increase in the rate of lipofuscin between various age groups.

further severe atrophy and shrunken appearance of neurons (Fig. 6Be1-3, f1-3). However, DG showed only mild degeneration in senile groups as well.

The striate cortex of 3-, 6-, and 12-month-old animals presented normal healthy neurons with distinct nucleus and nucleoli and brightly stained Nissl substance (Fig. 6Aa4, b4, c4). Degenerative changes in striate cortex appeared from 18 months in the form of shrunken appearance of 
neurons with pyknotic nuclei (Fig. 6Bd4). However, such cells with degenerative changes were much less even at 30 months of age than the cells in the hippocampus.

\section{Discussion}

The age-pigment, lipofuscin, accumulates in cells intrinsically and progressively with age. The process is more prominent in post-mitotic cells like neurons and reflects the chronological age of neuron. As large number of previous reports suggests that there is a variation in the rate of pigment accumulation among different species and, even with sex, age, and condition of the individual subject, the female rats used in this experiment were chosen from the same colony, maintained under same environmental conditions throughout their life span from birth till senescence.

The key role that hippocampus and the striate cortex play in cognition makes them critical targets to be studied in detail. The hippocampus is involved in learning and memory and most vulnerable to the process of ageing [43, 44]. Age-related accumulation of lipofuscin has been correlated with memory impairment [45]. Our study revealed region-specific accumulation of lipofuscin in the hippocampus. However, an almost similar pattern of lipofuscin accumulation was found all over the striate cortex. The present study indicates that the CA1 and CA3 subregions accumulated a higher amount of lipofuscin as compared to other subregions of hippocampus. Neurons of both CA 1 and CA3 subregions also showed severe neuronal atrophy in senile brains. However, the DG subregion showed minimum accumulation of lipofuscin and mild atrophy in neurons. This put forth the idea that the lipofuscin accumulation could be involved in the degeneration of neurons. The hippocampal CA3 region is most susceptible to age-related histopathological changes that account for cognitive decline during aging [44]. In agerelated neurodegenerative disorder like Alzheimer's disease, the prominent neuronal loss was observed in CA1 [46-49] and CA3 subregions [49] of hippocampus. Studies based on gene expression have well demonstrated that each hippocampal subregion has a distinct molecular profile that accounts for regional vulnerability [50-52].

In this study, we have made use of the autofluorescent property of the pigment in studying the possible accumulation of the age-pigment in the microglia with aging. The present study has revealed that the microglia especially the activated ones, in the hippocampus and striate cortex, accumulate distinct deposits of the pigment starting from 24 months of age onwards. Riew et al. [53] also reported

Lipofuscin in Aging Hippocampus and Striate Cortex

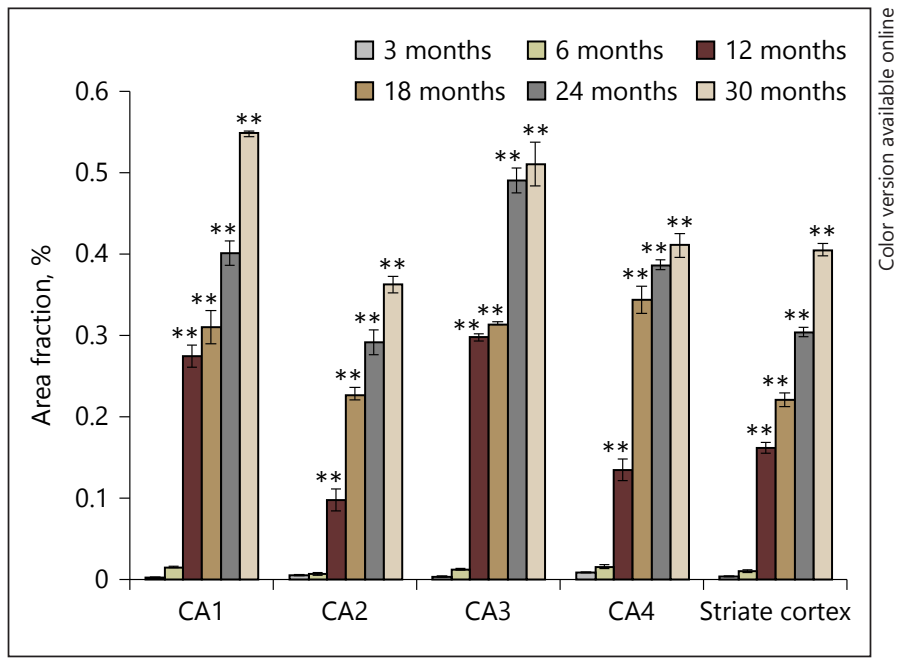

Fig. 5. Graph showing the comparison of percent area fraction of lipofuscin accumulation in various regions of hippocampus and striate cortex of different age groups of rats. Values are presented as mean $\pm \operatorname{SEM}(n=3) .{ }^{* *} p<0.001$ for comparison of the increase in the rate of lipofuscin between various age groups.

lipofuscin accumulation in brain macrophages. Lipofuscin aggregates, usually present golden-yellow fluorescence and rest in the close proximity of the nuclei. The fact that this pigment is in the microglia was confirmed with dual immunofluorescence labeling of neurons with NeuN, microglia with Iba1 and the autofluorescent nature of the pigment. The concept that microglia are known to play a role in removal of the age pigment from the senile neurons was forwarded by Hasan et al. [24] and Patro et al. [1]. Although lipofuscin research is focused on the presence and role of lipofuscin pigment in various cell types for more than a century, discrete descriptions on the presence of this pigment in the non-neuronal cells of the CNS continued to be debatable. In the present study, the presence of lipofuscin-laden microglia seen in close proximity of neurons and at site away from the neuron clearly indicates the presence of pigment in microglia. This suggests that there may be a transfer of pigment by exocytosis from neurons to microglia. Thus, in old age, macrophages become impaired with increased oxidative stress and lipofuscin accumulation, and show lower antioxidant defences [54]. This also indicates that in senile brains, microglia lose their ability to digest phagocytosed waste material, which appeared as lipofuscin deposits. This supports the idea that microglia that are unable to function properly contribute to immunosenescence in senile brain. The presence of lipofuscin-laden microglia located far from the neurons also suggests that microglia 


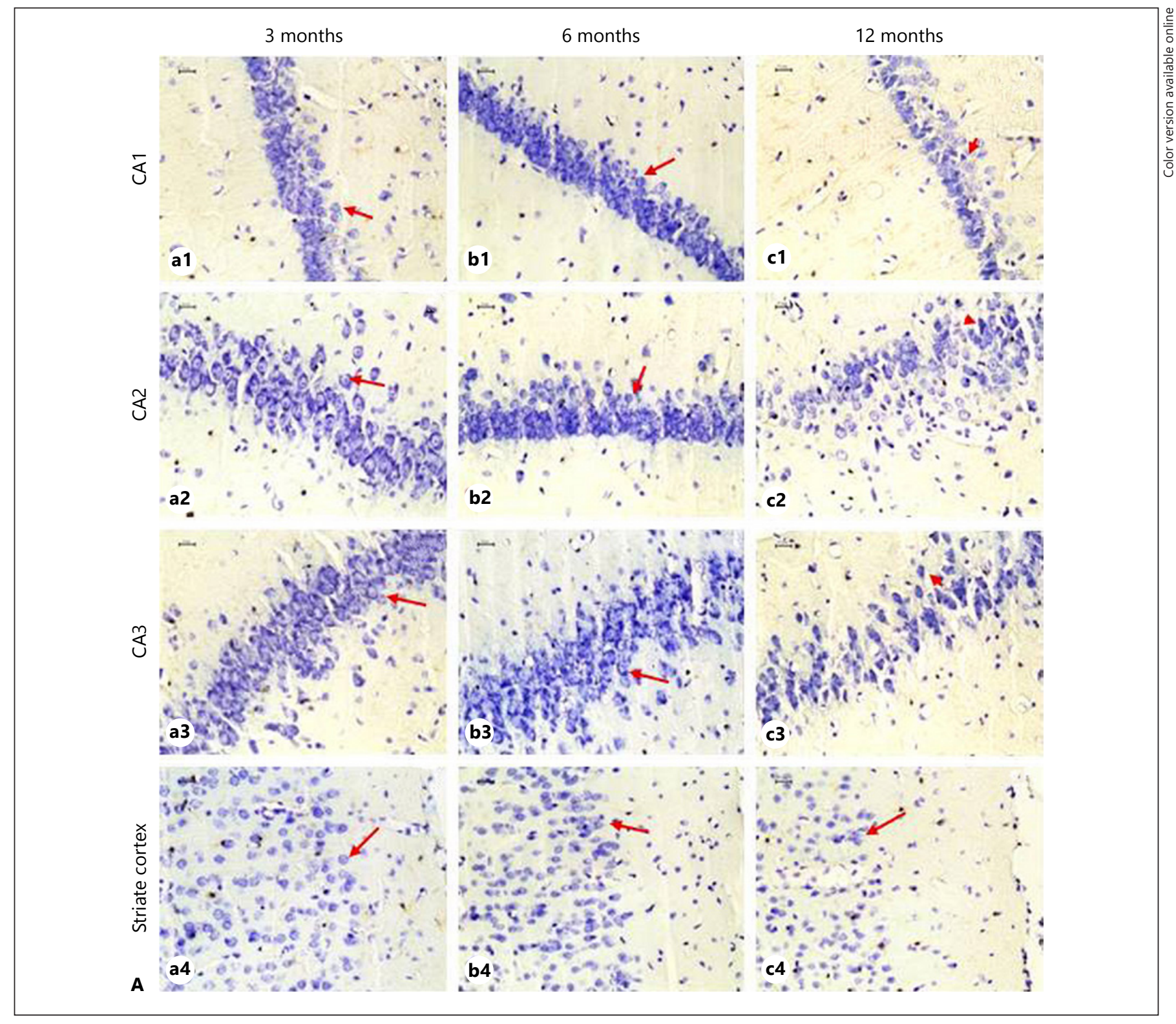

Fig. 6. A Light microscopic images of cresyl violet-stained sections showing the histological changes in hippocampal subregions and striate cortex of 3-, 6-, and 12-month-old rats. At 3 and 6 months of age, the neurons are densely packed in pyramidal cell layers of all the CA regions and at 3, 6, and 12 months in striate cortex with normal neuronal cell bodies with distinct nucleus and nucleolus (arrow). At

possibly accumulate waste product by themselves. The nondegradable accumulation of lipofuscin cause impairment of lysosomal function and phagocytic as well as autophagic alterations that may be hazardous to cell functions [55]. Braak et al. [29] also believed that an increasing lipofuscin accumulation leads to cell dysfunction and cell death. Thus, the progressive accumulation of lipofuscin
12 months of age, the atrophic neurons with shrunken appearance (arrowheads) were frequently seen in hippocampus. B Histological changes in cresyl violet-stained sections of hippocampal subregions and striate cortex of 18, 24, and 30 months. Chromatolysis was observed at 18 months (arrowhead) and atrophic neuronal bodies were frequently seen at 18, 24, and 30 months of age (arrow).

(For figure $6 b$ see next page.)

in neurons and microglia with age, as also reported in this study suggest its involvement in impairment of cellular function and homeostasis. A high amount of lipofuscin may cause oxidative injury to cells due to the impairment of lysosomal activity that in turn make them more vulnerable to oxidative stress [34]. Enhanced lipofuscin accumulation has also been correlated with increased oxida- 


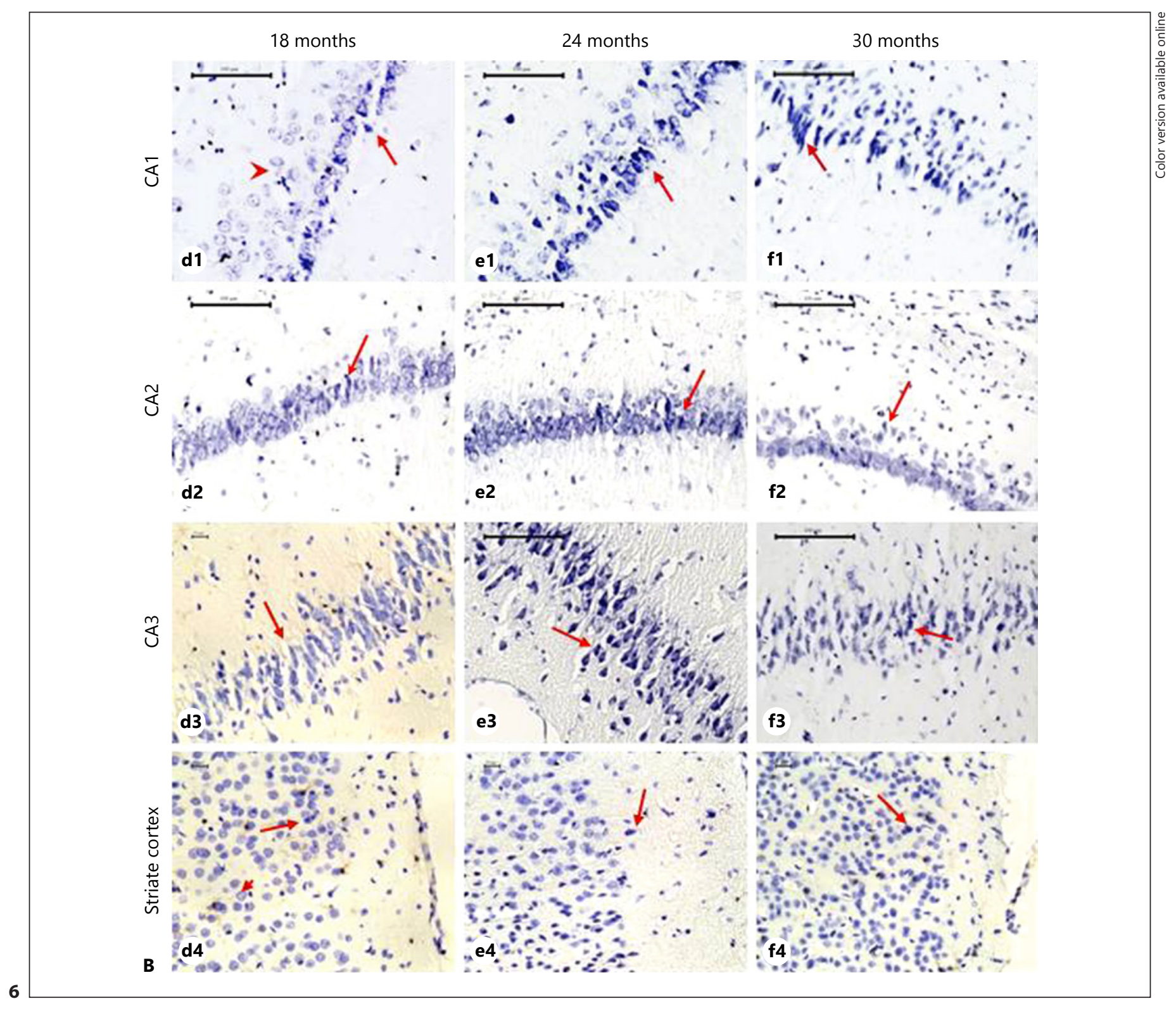

tive stress, decrease in antioxidative defence system, lysosomal iron overload, and mitochondrial dysfunction [27, 34]. Lipofuscin accumulation has also been linked with neuronal loss in normal aging process [56]. Excessive accumulation of lipofuscin ultimately decrease cellular adaptability and promote the development of age-related pathologies, viz., Alzheimer's disease, heart failure, frontal lobe epilepsy, and age-related macular degeneration [9, 57-61]. In addition, this higher age-related accumulation of lipofuscin could stimulate release of inflammatory chemokines and cytokines, which in turn lead to the activation of macrophage and microglia, and subsequently cause chronic inflammatory process [62].

Lipofuscin in Aging Hippocampus and Striate Cortex
In summary, aging contributes to intra-neuronal lipofuscin accumulation. Such deposits may interfere with neuronal function and contribute to age-related pathologies. The present study demonstrates that the CA 1 and CA 3 subfields are more prone to such age-associated changes. The main finding of this study is that, the microglia, which participates in the removal of the pigment from the neurons, themselves undergo the process of senescence and accumulate lipofuscin pigment. These senescent microglia may contribute to aging-related neurodegenerative conditions because they lose the neuroprotective potential, due to increased stress (oxidative stress) and may begin to over-secrete sub-

Ann Neurosci 2018;25:223-233

DOI: $10.1159 / 000490908$ 
stances that are harmful to the neurons and to the microglia themselves in addition to their decreased ability to effectively phagocytose insoluble materials including the age pigment.

\section{Acknowledgments}

The authors are thankful to Indian Council of Medical Research, New Delhi for financial assistance.

\section{Disclosure Statement}

The authors declare no conflict of interests.

\section{Author Contributions}

I.K.P. designed the study, S.S.K. performed the experiments and wrote the first draft, I.K.P. and N.P. analyzed the data and corrected the manuscript.

\section{References}

1 Patro IK, Sharma SP, Patro N: Neuronal lipofuscin: Its formation and reversibility. Ind Rev Life Sci 1988;8:95-120.

2 Patro N, Gupta P, Patro IK: Lipofuscin in cell aging and cell death. Indian J Gerontol 2002; 16:45-68.

3 Harman D: Lipofuscin and ceroid formation: the cellular recycling system. Adv Exp Med Biol 1989;266:3-15.

4 Ames BN, Shigenaga MK, Hagen TM: Oxidants, antioxidants, and the degenerative diseases of aging. Proc Natl Acad Sci U S A 1993; 90:7951-7922.

5 Beckman KB, Ames BN: The free radical theory of aging matures. Physiol Rev 1998;78:547581.

6 Porta EA: Pigments in aging: an overview. Ann N Y Acad Sci 2002;959:57-65.

7 Jolly RD, Douglas BV, Davey PM, Roiri JE: Lipofuscin in bovine muscle and brain: a model for studying age pigment. Gerontology 1995;41:283-295.

8 Jolly RD, Palmer DN, Dalefield RR: The analytical approach to the nature of lipofuscin (age pigment). Arch Gerontol Geriatr 2002; 34:205-217.

9 Terman A, Brunk UT: Molecules in focus Lipofuscin. Int J Biochem Cell Biol 2004;36:14001404.

10 Kikugawa K, Kato T, Beppu M, Hayasaka A: Fluorescent and cross-linked proteins formed by free radical and aldehyde species generated during lipid oxidation. Adv Exp Med Biol 1989;266:345-357.

11 Double KL, Dedov VN, Fedorow H, Kettle E, Halliday GM, Garner B, Brunk UT: The comparative biology of neuromelanin and lipofuscin in the human brain. Cell Mol Life Sci 2008; 10:7581-7589.

12 Terman A, Dalen H, Brunk UT: Ceroid/lipofuscin-loaded human fibroblasts show decreased survival time and diminished autophagocytosis during amino acid starvation. Exp Gerontol 1999;34:943-957.

13 Patro IK, Sharma SP, Patro N: Pre-lipofuscin: the concept and influence of centrophenoxine. Proc Nat Acad Sci (India) 1987;57:105-106.

14 Gilissen EP, Staneva-Dobrovski L: Distinct types of lipofuscin pigment in the hippocam- pus and cerebellum of aged cheirogaleid primates. Anat Rec 2013;296:1895-1906.

15 Nakano M, Oenzil F, Mizuno T, Gotoh S: Age-related changes in the lipofuscin accumulation of brain and heart. Gerentology 1995;41:69-79.

16 Brizzee KR, Ordy JM, Kaack B: Early appearance and regional differences in intraneuronal and extraneuronal lipofuscin accumulation with age in the brain of a nonhuman primate (Macaca mulatta). J Gerontol 1974; 29:366-381.

17 Oenzil F, Kishikawa M, Mizuno T, Nakano M: Age-related accumulation of lipofuscin in three different regions of rat brain. Mech Age Dev 1994;76:157-163.

18 Gerrits PO, Kortekaas R, de Weerd H, Veening JG, van der Want JJ: Regional differences in age-related lipofuscin accumulation in the female hamster brainstem. Neurobiol Aging 2012;33:625. e1-e9.

19 Sohal RS, Brunk UT: Lipofuscin as an indicator of oxidative stress and aging. Adv Exp Med Biol 1989;266:17-26.

20 Goyal VK: Lipofuscin pigment accumulation in human brain during aging. Exp Gerontol 1982;17:481-487.

21 Patro N, Sharma SP, Patro IK: Lipofuscin accumulation in ageing myocardium and its removal by meclophenoxate. Ind J Med Res 1992;96:192-198.

22 Terman A, Brunk UT: Oxidative stress, accumulation of biological "garbage" and aging. Antioxid Redox Signal 2006;8:197-204.

23 Zivkovic V, Stefanovic N, Durovic-Filipovic T, Pavlovic S, Stojanovic V, Bakic M, Kundalic B, Pavlovic M: Patterns of lipofuscin accumulation in ganglionic nerve cells of superior cervical ganglion in humans. Vojnosanit Pregl 2008;65:738-742.

24 Hasan M, Glees P, Spoerri PE: Dissolution and removal of neuronal lipofuscin following dimethyl-aminoethyl p-cholorophenoxyacetate administration to guinea pigs. Cell Tiss Res 1974;150:369-375.

25 Benavides SH, Monserrat AJ, Farina S, Porta EA: Sequential histochemical studies of neuronal lipofuscin in human cerebral cortex from the first to the ninth decade of life. Arch Gerrontol Geriatr 2002;34:219231.

26 Fonseca DB, Sheehy MR, Blackman N, Shelton PM, Prior AE: Reversal of a hallmark of brain ageing: lipofuscin accumulation. Neurobiol Aging 2004;26:69-76.

27 Riga D, Riga S, Halalau F, Schneider F: Brain lipopigment accumulation in normal and pathological aging. Ann N Y Acad Sci 2006; 1067:158-163.

28 Sharma SP, Manocha SL: Lipofuscin formation in the developing nervous system of squirrel monkeys consequent to maternal dietary protein deficiency during gestation. Mech Age Dev 1977;6:1-14.

29 Braak H, Braak E, Gullotta F, Goebel HH: Pigment-filled appendages of the small spiny neurons: a severe pathological change of the striatum in neuronal ceroid lipofuscinosis. Neuropathol Appl Neurobiol 1979;5:389394.

30 Braak H, Braak E: Neuronal types in the striatum of man. Cell tissue Res 1982;227:319342 .

31 Tigges J, Herndon JG, Peters A: Neuronal population of area 4 during the life span of the rhesus monkey. Neurobiol Aging 1990; 11:201-208.

$32 \mathrm{Xu} \mathrm{H}$, Chen M, Manivannan A, Lois N, Forrester JV: Age-dependent accumulation of lipofuscin in perivascular and subretinal microglia in experimental mice. Ageing Cell 2008;7:58-68.

33 Glees P, Hasan M: Intracytoplasmic location of lipofuscin granules. In: Lipofuscin in Neuronal Ageing and Disease; in Bergmann W, Doerr W (eds): Advances in Anatomy and Pathology, Georg Thieme Verlag, Stuttgart, 1976, vol 32, pp 10-17.

34 Brunk UT, Terman A: The mitochondrial-lysosomal axis theory of aging: accumulation of damaged mitochondria as a result of imperfect autophagocytosis. Eur J Biochem 2002; 269:1996-2002.

35 Elleder M, Sokolová J, Hrebícek M: Follow-up study of subunit c of mitochondrial ATP synthase (SCMAS) in Batten disease and in unrelated lysosomal disorders. Acta Neuropathol 1997;4:374-90. 
36 Koniga J, Ott C, Hugo M, Jung T, Bulteau AL, Grune T, Hohn A: Mitochondrial contribution to lipofuscin formation. Redox Biol 2017; 11:673-681.

37 Mann DM, Yates PO: Lipoprotein pigments - their relationship to ageing in the human nervous system. II. The melanin content of pigmented nerve cells. Brain 1974;97, 489498.

38 Mann DMA, Yates PO: Ageing, nucleic acids and pigments; in Smith W, Thomas, Cavanagh JB (eds): Recent Advances in Neuropathology. Edinburgh, Churchill Livingstone, 1982, vol 2, pp 109-138.

39 Brody $\mathrm{H}$ : The deposition of aging pigment in the human cerebral cortex. J Gerontol 1960; 15:258-261.

$40 \mathrm{Braak}$ H: On the striate area of the human isocortex. A Golgi- and pigmetarchitectonic study. J comp Neurol 1976;166:341-364.

41 Peters A, Sethares C: Aging and the Meynert cells in rhesus monkey primary visual cortex. Anal Rec 1993;236:721-729.

42 Humason GL: Animal Tissue Techniques; in Beadle GW, Emerson R, Whitaker DW (eds): W H Freeman and company, San Francisco and London, 1962, pp 195-196.

43 Miller DB, O'Callaghan JP: Aging, stress and the hippocampus. Ageing Res Rev 2005;4:123140.

44 Bartsch T, Wulff P: The hippocampus in aging and disease: from plasticity to vulnerability. Neuroscience 2015;309:1-16.

45 Asuncao M, Santos-Marques MJ, Carvalho F, Lukoyanov NV, Andrade JP: Chronic green tea consumption prevent age-related changes in rat hippocampal formation. Neurobiol Aging 2011;32:707-717.
46 Kadar T, Silbermann M, Brandeis R, Levy A: Age-related structural changes in the rat hippocampus: correlation with working memory deficiency. Brain Res 1990;512:113-120.

47 West MJ, Coleman PD, Flood DG, Troncoso JC: Differences in the pattern of hippocampal neuronal loss in normal ageing and Alzheimer's disease. Lancet 1994;344:769-772.

48 Lucassen PJ, Heine VM, Muller MB, Van der Beek EM, Wiegant VM, De Kloet ER, Joels M, Fuchs E, Swaab DF, Czeh B: Stress, depression and hippocampal apoptosis. CNS Neurol Disord Dru Targets 2006;5:531-546.

49 Padurariu M, Ciobica A, Mavroudis I, Fotiou D, Baloyannis S: Hippocampal neuronal loss in the CA1 and CA3 areas of Alzheimer's disease patients. Psychiatria Danubina 2012;24: 152-158.

50 Zhao X, Lein ES, He A, Smith SC, Aston C, Gage FH: Transcriptional profiling reveals strict boundaries between hippocampal subregions. J Comp Neurol 2001;441:187-196.

51 Thompson CL, Pathak SD, Jeromin A, Ng LL, MacPherson CR, Mortrud MT, Cusick A, Riley ZL, Sunkin SM, Bernard A, Puchalski RB, Gage FH, Jones AR, Bajic VB, Hawrylycz MJ, Lein ES: Genomic anatomy of the hippocampus. Neuron 2008;60:1010-1021.

52 Fanselow MS, Dong HW: Are the dorsal and ventral hippocampus functionally distinct structures? Neuron 2010;65:7-19.

53 Riew TR, Kim HL, Choi JH, Jin X, Shin YJ, Lee MY: Progressive accumulation of autofluorescent granules in macrophages in rat striatum after systemic 3-nitropropionic acid: a correlative light- and electron-microscopic study. Histochem Cell Biol 2017;148:517528
54 Vida C, de Toda IM, Cruces J, Garrido A, Gonzalez-Sanchez M, De La Fuente M: Role of macrophages in age-related oxidative stress and lipofuscin accumulation in mice. Redox Biol 2017;12:423-437.

55 Brunk UT, Terman A: Lipofuscin: Mechanisms of age-related accumulation and influence on cell function. Free Radic Biol Med 2002;33:611-619.

56 Mestre N, Bons N: Age-related cytological changes and neuronal loss in basal forebrain cholinergic neurons in Microcebus murinus (lemurian, primate). Neurodegeneration 1993;2:25-32.

57 Dorey CK, Wu G, Ebenstein D, Garsd A, Weiter JJ: Cell loss in the aging retina. Relationship to lipofuscin accumulation and macular degeneration. Invest Ophthalmol Vis Sci 1989;30:1691-1699.

58 Dowson JH, Mountjoy CQ, Cairns MR, Wilton-Cox H: Changes in intraneuronal lipopigment in Alzheimer's disease. Neurobiol Aging 1992;13:493-500.

59 Wolf G: Lipofuscin and macular degeneration. Nutr Rev 2003;61:342-346.

60 Sparrow JR, Boulton M: RPE lipofuscin and its role in retinal pathobiology. Exp Eye Res 2005;80:595-606.

61 Liu JY, Reeves C, Diehl B, Coppola A, AlHajri A, Hoskote C, Mughairy SA, Tachrount M, Groves M, Michalak Z, Mills K, McEvoy AW, Miserocchi A, Sisodiya SM, Thom M: Early lipofuscin accumulation in frontal lobe epilepsy. Ann Neurol 2016;80:882-895.

62 Anderson OA, Finkelstein A, Shima DT: A2E induces IL-1ß production in retinal pigment epithelial cells via the NLRP3 Inflammasome. PLoS One 2013;8:e67263. 